\title{
Experiencia diagnóstica de diez años del tumor neuroendocrino de pulmón en la Fundación Santa Fe de Bogotá: 2001 - 2010
}

\section{Ten years of experience in diagnosing neuroendocrine neoplasms of the lung at the Fundación Santa Fe de Bogotá: 2001 - 2010}

Lucia Beatriz Taboada Barrios, MD. (1); Rocio del Pilar López Panqueva, MD. (2); Luis Gerardo Garcia-Herreros Hellal, MD. ${ }^{(3) ;}$

Deyanira González Devia, MD.(4)

\section{RESUMEN}

Introducción: los tumores neuroendocrinos de pulmón surgen a partir de las células de Kuichitsky dispersas en todo el árbol bronquial; éstos tienen un amplio espectro de diferenciación histológica y son capaces de producir aminas bioactivas.

Objetivos: describir las características clinicopatológicas de la población con diagnóstico de tumores neuroendocrinos de pulmón en el Hospital Universitario Fundación Santa Fe de Bogotá (HUFSFB) de 2001 a 2010.

Diseño: estudio descriptivo tipo corte transversal.

Materiales y métodos: se incluyó toda la población con tumores neuroendocrinos de pulmón confirmados por patología en el HUFSFB durante el periodo 2001 a 2010. Se revisaron los reportes de patologías y los registros médicos para la obtención de datos.

Resultados: se incluyeron 132 casos. El tipo de tumor más frecuente fue el carcinoma de célula pequeña. El carcinoide
tipico fue cinco veces más frecuente que el atípico. Los tumores bien diferenciados fueron más frecuentes en mujeres y
los de alto grado en hombres, presentándose a mayor edad y asociados al antecedente de tabaquismo. La principal forma
de llegar al diagnóstico fue la aparición de sintomas secundarios al tumor. De todos los sujetos, $50 \%$ tenia metástasis al
momento del diagnóstico, la mayoría en las formas mal diferenciadas.

Conclusiones: el pulmón presenta un espectro histológico, clínico y bioquímico muy heterogéneo de tumores neuroendocrinos. Aun no hay datos nacionales de incidencia y supervivencia de este tipo de tumor. Se busca motivar la investigación en este campo y alertar sobre el seguimiento necesario, incluso en los tumores bien diferenciados.

Palabras clave: cáncer de pulmón, tumores neuroendocrinos, carcinoma neuroendocrino, tumor carcinoide, carcinoma de células pequeñas del pulmón.

\section{ABSTRACT}

Introduction: neuroendocrine tumors (NET) of the lung arise from Kulchitsky cells, which are found throughout the bronchial tree. These tumors have a broad range of histologic differentiation, and are capable of producing bioactive amines.

Departamento de Medicina Interna, Sección Endocrinología, Hospital Universitario Fundación Santa Fe de Bogotá. Bogotá. Colombia.

(1) Servicio Social Obligatorio, Medicina Interna, Fundación Santa Fe de Bogotá.

(2) Departamento de Patologia y Laboratorios, Hospital Universitario Fundación Santa Fe de Bogotá. Profesor Facultad de Medicina, Universidad de los Andes. Bogotá, Colombia.

(3) Cirugia de Tórax, Hospital Universitario Fundación Santa Fe de Bogotá. Profesor Facultad de Medicina, Universidad de los Andes. Bogotá, Colombia.

(4) Medicina Interna, Endocrinología Oncológica, Hospital Universitario Fundación Santa Fe de Bogotá. Profesor Facultad de Medicina, Universidad de los Andes. Bogotá, Colombia.

Correspondencia: Dra. Deyanira González Devia. Calle 119 No. 7 - 75. $2^{\circ}$ Piso. Clínicas especializadas. Hospital Universitario Fundación Santa Fe de Bogotá. Teléfono: (571) 6030303 Ext. 5069. Correo electrónico: deyaniragonzalezdevia@yahoo.com, luciataboadab@gmail.com

Recibido: agosto 5 de 2012. Aceptado: noviembre 12 de 2012. 
Objectives: to describe the clinical and pathological characteristics of the population diagnosed with NET of the lung at the Hospital Universitario Fundación Santa Fe de Bogotá (HUFSFB) between the years 2001 and 2010.

Design: descriptive cross-cut study.

Materials and methods: the entire population with confirmed NET of the lung at the HUFSB over the period 2001-2010 was included. Pathology reports and medical charts were reviewed for obtaining data.

Results: one hundred and thirty two (132) cases were included. The most common type of tumor was small-cell lung carcinoma. Typical carcinoid was five-fold more frequent than the atypical. Well-differentiated tumors were more common in women, and high-grade tumors were more common in men. High-grade tumors presented at more advanced ages and were associated with smoking. The main path for reaching diagnosis was the appearance of symptoms due to the tumor. Of all subjects, $50 \%$ had metastatic disease at the moment of diagnosis, mostly in poorly-differentiated types.

Conclusions: the lung has a very heterogeneous spectrum of histological, clinical, and biochemical types of NETs. There are still no national data with regard to incidence and survival of this type of tumor. We seek to motivate research in this field and to call attention to the need of follow-up in these cases, including well-differentiated tumors.

Keywords: lung cancer, neuroendocrine tumors, neuroendocrine carcinoma, carcinoid tumor, small-cell carcinoma of the lung.

Rev Colomb Neumol 2013; 24 (2):84-91

\section{INTRODUCCIÓN}

Los tumores neuroendocrinos son un grupo de neoplasias complejas originadas en el tejido endocrino, cuyas células pueden estar organizadas en glándulas o existir como parte de un sistema difuso compuesto por las células argentafines localizadas principalmente en el epitelio intestinal y respiratorio (1). En el pulmón estos tumores surgen a partir de células neuroendocrinas llamadas células de Kulchitsky dispersas de manera difusa en todo el árbol bronquial y capaces de producir aminas bioactivas como hormona adrenocorticotropa (ACTH), hormona antidiurética $(A D H)$, hormona de crecimiento $(G H)$ y serotonina, entre otras (2).

Estos tumores, al igual que otros tumores neuroendocrinos, tienen un amplio espectro de diferenciación, tanto desde el punto de vista molecular como morfológico, que varia desde las formas bien diferenciadas, incluyendo el carcinoide típico (CT) de bajo grado y el carcinoide atipico (CA) de grado intermedio (grados | y || respectivamente), hasta las formas pobremente diferenciadas constituidas por el carcinoma de células pequeñas (CCP) y el carcinoma neuroendocrino de células grandes (CNECG), considerados de alto grado (grado III) $(3,4)$. De acuerdo con la última clasificación de la Organización Mundial de la Salud (OMS) de 2004 (5), los tumores neuroendocrinos de pulmón se diagnostican y se clasifican según se observa en la tabla 1.

Dentro del espectro de proliferación de las células neuroendocrinas de pulmón se destacan otras variantes histológicas como la hiperplasia de células neuroendocrinas, que no sobrepasa la membrana basal, los tumorlets que son proliferaciones nodulares de células neuroendocrinas con morfología similar a la de un tumor neuroendocrino carcinoide típico, pero con un diámetro máximo de $5 \mathrm{~mm}$ y que pueden sobrepasar la membrana basal, y la hiperplasia difusa idiopática de células neuroendocrinas pulmonares, la

Tabla 1. Clasificación de los tumores neuroendocrinos de pulmón de la OMS 2004.

\begin{tabular}{|c|c|c|c|c|}
\hline \multicolumn{5}{|c|}{$\begin{array}{l}\text { Clasificación histológica de los tumores neuroendocrinos de pulmón } \\
\text { Criterios de la OMS }\end{array}$} \\
\hline & CT & $\mathrm{CA}$ & CNECG & $\mathrm{CCP}$ \\
\hline Diferenciación & Bien diferenciado & Bien diferenciado & Mal diferenciado & Mal diferenciado \\
\hline Mitosis (x10 CAP) & $<2$ & $2-10$ & $>10$ (Promedio 70) & $>10$ (Promedio 80) \\
\hline Necrosis & Ausente & Usualmente focal & Extensa & Extensa \\
\hline
\end{tabular}

Adaptada de: Rekhtman N. Neuroendocrine tumors of the lung: an update. Arch Pathol Lab Med. 2010; 134 (11): 1628-38. 
cual es una condición muy rara que representa una lesión preneoplásica de un TC dada su asociación con fibrosis e inflamación en sujetos que tienen diez o más lesiones neuroendocrinas de pulmón $(6,7)$. Por último, están los tumores mixtos en los que el componente neuroendocrino se encuentra en diferentes proporciones, con alguno de los carcinomas del tipo célula no pequeña (8).

Los tumores neuroendocrinos de pulmón corresponden aproximadamente al 25\% (22-27\%) de los tumores neuroendocrinos del sistema endocrino difuso $(9,10)$, con una incidencia anual estimada de 1,35 por 100.000 personas al año (11); en Colombia no se tienen datos publicados para esta patología.

En este estudio se describen las características clinicopatológicas de la población con diagnóstico de tumores neuroendocrinos de pulmón del Hospital Universitario Fundación Santa Fe de Bogotá (HUFSFB) en el periodo de 2001 a 2010.

\section{METODOLOGÍA}

Se realizó un estudio descriptivo de tipo corte transversal en el momento del diagnóstico, que incluyó toda la población con tumores neuroendocrinos de pulmón confirmados por patologia en el HUFSFB durante el periodo 2001 a 2010. Se hizo una búsqueda electrónica en los archivos de patología y una revisión del registro médico correspondiente con el fin de obtener los datos clínicos de los sujetos.

Se analizaron las siguientes variables: edad y género, grado histológico, presencia y sitio de metástasis al momento del diagnóstico, forma de llegar al diagnóstico y manifestaciones clínicas asociadas. Para las variables que requerian acceso a la historia clínica se incluyeron únicamente los sujetos con registro dentro de los archivos del HUFSFB.

Este trabajo fue aprobado por el comité de Ética Institucional.

El análisis estadístico se realizó con Stata 12 y Excel 2010. Se calcularon medidas de tendencia central, frecuencia simple, rangos y porcentajes.

\section{RESULTADOS}

Se encontraron en total 139 sujetos con reportes de patologías confirmatorias de neoplasia neuroendocrina de pulmón; para el análisis se excluyeron siete casos con diagnóstico de tumorlet, debido a que no hacen parte de la clasificación de tumores neuroen- docrinos propuesta por la OMS en la actualidad, y en su mayoría son hallazgos quirúrgicos incidentales. $\mathrm{Al}$ final, se incluyeron en total 132 casos.

\section{Frecuencia por año}

La frecuencia anual tuvo un aumento notorio desde el año 2003 con 16 casos, sin haber grandes variaciones en años posteriores. En 2001 se notó una frecuencia mucho menor a la encontrada en los demás años, con únicamente dos casos diagnosticados. El comportamiento fue similar para ambos grupos de tumores, siendo los mal diferenciados más frecuentes en la mayoria de los años, excepto en 2003 (Figura 1).

\section{Presentación histológica}

El tipo de tumor más frecuente fue el CCP correspondiendo a más del $50 \%$ de los casos. Seis casos fueron no clasificados: un caso por material insuficiente para diferenciar entre típico y atípico, y el resto clasificados como mal diferenciados sin especificar el tamaño celular (Tabla 2).

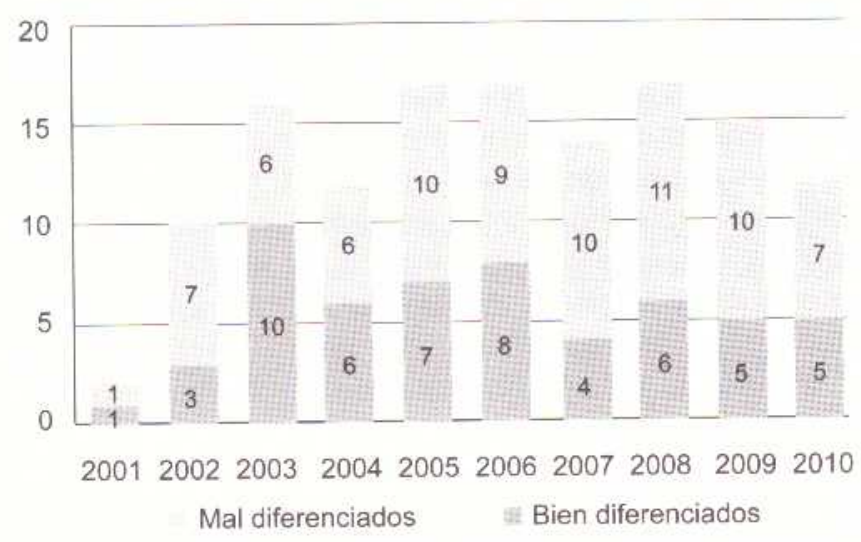

Figura 1. Frecuencia anual de tumores neuroendocrinos de pulmón.

Tabla 2. Frecuencia de tumores de acuerdo con la clasificación histológica.

\begin{tabular}{|lll|}
\hline \multicolumn{2}{l|}{ Clasificación histológica } & \\
\hline & $(\boldsymbol{n})$ & Porcentaje \\
Carcinoide típico & 46 & 34,9 \\
Carcinoide atipico & 9 & 6,8 \\
CCP & 69 & 52,3 \\
CNECG & 2 & 1,5 \\
No clasificado & 6 & 4,5 \\
Total & 132 & 100 \\
\hline
\end{tabular}


De las formas bien diferenciadas hay 55 casos de tumores carcinoides; $83,6 \%$ fueron CT y $16,4 \%$ CA.

\section{Perfil demográfico de acuerdo con el tipo de tumor}

En general los tumores neuroendocrinos de pulmón fueron más frecuentes en mujeres $(53 \%$; 66 de 126 casos) con una edad promedio de presentación de 61,3 años (rango 11 - 82) y mediana de 65 años.

De acuerdo con el grado histológico, los tumores bien diferenciados fueron más frecuentes en mujeres, con $80,4 \%$ y $66,7 \%$ respectivamente para el CT y CA; mientras que los tumores neuroendocrinos de alto grado fueron más frecuentes en hombres y con una edad de presentación más tardia. El antecedente de tabaquismo fue analizado en 40 sujetos; $87,5 \%$ de los CCP fueron fumadores en comparación con $35 \%$ de los CT, si se tienen en cuenta únicamente los sujetos con datos (Tabla 3).

\section{Síntomas y forma de llegar al diagnóstico}

Para el diagnóstico se cuenta con 45 registros completos, se excluye uno correspondiente a un tumor no clasificado. La principal forma de llegar al diagnóstico fue por la aparición de síntomas relacionados con el tumor y en segundo lugar por los hallazgos radiológicos incidentales.
Entre los pacientes que debutaron con sintomatologia la principal manifestación fueron sintomas respiratorios que incluyeron tos, hemoptisis, neumonia obstructiva, disnea y dolor pleuritico. Otros síntomas menos frecuentes fueron dolor crónico relacionado con el tumor, síndrome de vena cava superior, SIADH, acromegalia y alteraciones neurológicas o sindrome medular como sintomatología de metástasis al sistema nervioso. Cabe aclarar que varios pacientes debutaron con más de un sintoma (Tabla 4).

\section{Presencia de metástasis}

Se analizaron 90 casos para metástasis; de todos los sujetos con datos $71 \%$ tenía metástasis al momento del diagnóstico.

En los tumores bien diferenciados, el CT presenta en $11 \%$ invasión a nivel de ganglios regionales, mientras que el CA lo hace en $62,5 \%$ de los casos tanto a nivel ganglionar regional como a distancia. Para el CCP, $98 \%$ tenía metástasis a distancia. El principal sitio de metástasis a distancia fue el hígado (Tabla 5, figura 2).

\section{DISCUSIÓN}

La incidencia y prevalencia del tumor neuroendocrino de pulmón se considera subestimada debido a la pobre correlación con la mortalidad, al comportamiento indolente del tumor neuroendocrino bien

Tabla 3. Resumen de las características demográficas de acuerdo con el tipo de tumor.

\begin{tabular}{|c|c|c|c|c|}
\hline \multicolumn{5}{|c|}{ Características demográficas de acuerdo con el tipo de tumor } \\
\hline & $\begin{array}{l}\text { CT } \\
n=46\end{array}$ & $\begin{array}{l}\text { CA } \\
n=9\end{array}$ & $\begin{array}{l}\text { CCP } \\
n=69\end{array}$ & $\begin{array}{l}\text { CNECG } \\
n=2\end{array}$ \\
\hline \multicolumn{5}{|l|}{ Género } \\
\hline $\begin{array}{l}\text { Masculino } \\
n_{1}(\%)\end{array}$ & $9(19,6)$ & $3(33,3)$ & $46(66,7)$ & $2(100)$ \\
\hline $\begin{array}{l}\text { Femenino } \\
\mathrm{n},(\%)\end{array}$ & $37(80,4)$ & $6(66,7)$ & $23(33,3)$ & $0(0)$ \\
\hline Razón M:F & $1: 4$ & $1: 2$ & $2: 1$ & N/A \\
\hline \multicolumn{5}{|l|}{ Edad (años) } \\
\hline Promedio & 56,6 & 62,9 & 64,5 & 66,5 \\
\hline Rango & $11-81$ & $46-75$ & $38-82$ & $65-68$ \\
\hline \multicolumn{5}{|c|}{ Antecedente de tabaquismo } \\
\hline $\begin{array}{l}\mathrm{Si} \\
\mathrm{N}(\%)\end{array}$ & $7(15,2)$ & $3(33,3)$ & $14(20,3)$ & 0 \\
\hline $\begin{array}{l}\text { No } \\
\text { N (\%) }\end{array}$ & $13(28,3)$ & $1(11,1)$ & $2(2,9)$ & 0 \\
\hline $\begin{array}{l}\text { No dato } \\
n(\%)\end{array}$ & $26(56,5)$ & $5(55,6)$ & $53(76,8)$ & $2(100)$ \\
\hline
\end{tabular}


Tabla 4. Forma de llegar al diagnóstico y sintomas asociados a cada tipo de tumor.

\begin{tabular}{|lccccc|}
\hline \multicolumn{7}{l}{ Forma de diagnóstico y sintomas de acuerdo con el tipo de tumor } & & \\
\hline & Total & CT & CA & CCP & CNCG \\
\hline Forma de llegar al diagnóstico $n=44$ & & & & & \\
$\quad$ Manifestaciones clínicas & $29(65,9)$ & 13 & 3 & 13 & 0 \\
Hallazgo radiológico & $15(34,1)$ & 12 & 2 & 1 & 0 \\
Clínica predominante & & & & & \\
Síntomas respiratorios & 23 & 12 & 2 & 9 & 0 \\
Alteraciones neurológicas/paraplejía & 2 & 0 & 1 & 1 & 0 \\
Dolor crónico relacionado con el tumor & 3 & 0 & 0 & 3 & 0 \\
Pérdida de peso & 2 & 0 & 0 & 2 & 0 \\
Síndrome de vena cava & 2 & 0 & 0 & 2 & 0 \\
Acromegalia & 1 & 1 & 0 & 0 & 0 \\
SIADH & 1 & 0 & 0 & 1 & 0 \\
\hline
\end{tabular}

Tabla 5. Presencia de metástasis de acuerdo con el tipo de tumor.

\begin{tabular}{|c|c|c|c|c|}
\hline & $\begin{array}{l}\text { CT } \\
n=46\end{array}$ & $\begin{array}{l}C A \\
n=9\end{array}$ & $\begin{array}{l}\text { CPP } \\
n=69\end{array}$ & $\begin{array}{l}\text { CNCG } \\
n=2\end{array}$ \\
\hline \multicolumn{5}{|c|}{ Metástasis al diagnóstico } \\
\hline $\begin{array}{l}\mathrm{Si} \\
\mathrm{n}(\%)\end{array}$ & $3(6,5)$ & $5(55,6)$ & $53(76,8)$ & $1(50)$ \\
\hline Ganglios regionales & 3. & 2 & 17 & 0 \\
\hline A distancia & 0 & 3 & 35 & 1 \\
\hline No dato & 0 & 0 & 1 & 0 \\
\hline $\begin{array}{l}\text { No } \\
n(\%)\end{array}$ & $24(52,2)$ & $3(33,3)$ & $1(1,5)$ & 0 \\
\hline $\begin{array}{l}\text { No dato } \\
\mathrm{n}(\%)\end{array}$ & $19(41,3)$ & $1(11,1)$ & $15(21,7)$ & $1(50)$ \\
\hline
\end{tabular}

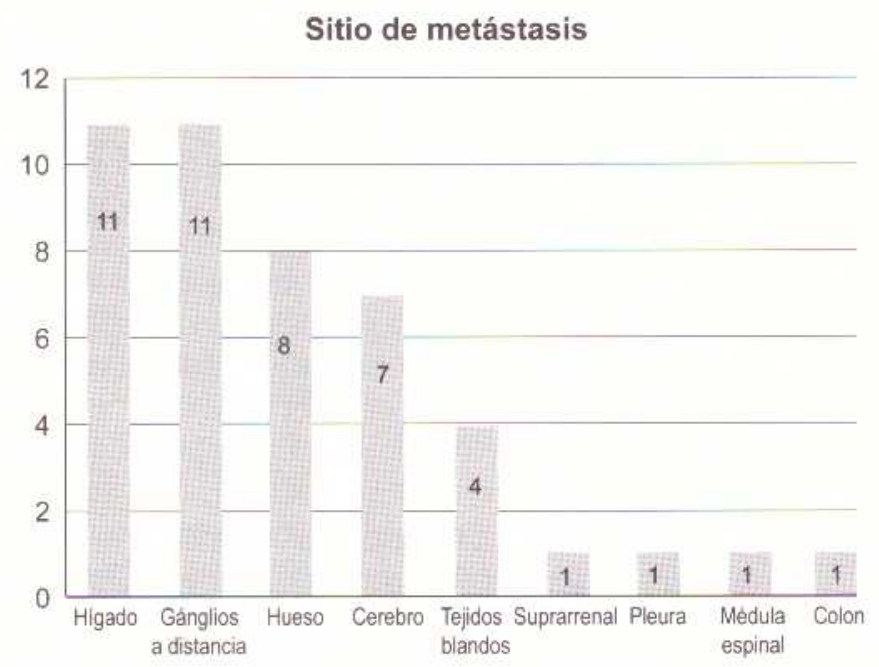

Figura 2. Frecuencia de sitios de metástasis a distancia. diferenciado y a la falta de asociación entre los signos clinicos y la gravedad de la enfermedad; por tanto, algunos tumores neuroendocrinos bien diferenciados nunca se detectarán (12). A pesar de esto, en los últimos años se ha documentado incremento en la incidencia. La base de datos publicada por Modlin y colaboradores (9), incluye el análisis de 13.715 sujetos con tumores carcinoides de 1950 a 1999 , de los cuales $3.365(24,5 \%)$ casos correspondian a tumores neuroendocrinos bien diferenciados típicos y atípicos traqueobronquiales. Desde 1950 a 1999 la incidencia de estos tumores aumentó de 0,22 a 0,52 x 100.000 habitantes en hombres blancos y de 0,24 a $0,89 x$ 100.000 habitantes en mujeres blancas, al igual que el porcentaje de tumores neuroendocrinos correspondientes al pulmón, que ha pasado de $10,2 \%$ a $27,9 \%$. Es preciso tener en cuenta que esta incidencia 
incluye únicamente a las formas bien diferenciadas. De igual forma, un estudio realizado en Dinamarca mostró un aumento en los tumores neuroendocrinos de pulmón diferente a carcinoma de célula pequeña de 0,24 a 0,53 por 100.000 habitantes-año en hombres y de 0,14 a 0,41 por 100.000 habitantes-año en mujeres desde 1978 a 1997 (13).

El estudio que aquí se publica no busca estimar la incidencia de los tumores neuroendocrinos de pulmón, dada la naturaleza del mismo; sin embargo, se puede ver un leve aumento en la frecuencia de tumores neuroendocrinos de pulmón con el paso de los años. El aumento en la frecuencia en el HUFSF, como en el mundo, probablemente se relaciona con la evolución de las técnicas diagnósticas, el incremento de la utilización de imágenes radiológicas y el acceso a los servicio médicos, factores que pueden influir en su epidemiología $(6,12)$. El aumento notorio de la frecuencia después del año 2001, probablemente sea el resultado de la creación de un grupo multidisciplinario y la difusión del conocimiento de la enfermedad, que han permitido ser reconocidos como un centro de referencia para el estudio de este tipo de lesiones.

Las formas histológicas bien diferenciadas son infrecuentes; el CT corresponde al 1\%-2\% de las malignidades de pulmón y al 85 a $90 \%$ de los bien diferenciados y el CA a sólo el 0,1\%-0,2\% de las malignidades de pulmón y a menos del $10 \%$ de los carcinoides de pulmón $(3,14,15)$. En nuestra serie los carcinoides presentan una distribución similar, siendo los CT cinco veces más frecuentes que el CA.

Las formas mal diferenciadas son más frecuentes; el CCP corresponde al $15 \%$ a $20 \%$ de las malignidades invasivas de pulmón, y el CNECG al $3 \%$ del total de cánceres de pulmón (16). En nuestra serie el CCP corresponde a más de la mitad de los tumores neuroendocrinos de pulmón y fue mucho más frecuente que el CNECG, similar a lo reportado en otras series (13). Una posible explicación de la mayor frecuencia de las formas mal diferenciadas es su curso más agresivo y la presencia de sintomas que motivan el diagnóstico diferencial, contrario de los bien diferenciados que pueden ser asintomáticos, diagnosticándose algunos como hallazgos incidentales y algunos incluso nunca diagnosticarse.

Los tumores carcinoides de pulmón (CT y CA) tienen una edad promedio de presentación de 45 a 55 años; no hay una predilección clara por algún género, aunque en sujetos menores de 50 años hay predilección por el femenino, y no hay relación con el antecedente de tabaquismo $(16,17)$. Por su parte, los carcinomas mal diferenciados tienden a ocurrir en edades mayores, con una media de presentación de 60 a 70 años para el CCP y 62 años para el CNECG, con predominio por el género masculino, reportado en series hasta 8 a 9 veces más frecuente en hombres y en donde el antecedente de tabaquismo está presente en la mayoría de los pacientes $(12,16,18,19)$. En nuestra serie los hallazgos se correlacionan con lo reportado en la literatura, con un promedio de edad creciente a lo largo del espectro de diferenciación, que va de 56,4 y 62,9 años respectivamente para las formas bien diferenciadas, carcinoide típico y atípico, hasta 64,5 y 66,5 años para las formas mal diferenciadas. La diferencia de género es evidente, presentándose las formas bien diferenciadas con mayor frecuencia en el género femenino contrario de lo reportado en la literatura donde se aprecia una distribución similar de acuerdo con el género, y las formas mal diferenciadas con mayor frecuencia en el género masculino, lo que esté probablemente relacionado con la mayor exposición al tabaquismo en este género.

La presentación clínica de los tumores neuroendocrinos depende del grado histológico tumoral, del sitio de la lesión, de la producción hormonal, del compromiso o extensión tumoral y de la presencia de un síndrome heredofamiliar. Aproximadamente la mitad de los sujetos con tumores neuroendocrinos bien diferenciados son asintomáticos al momento del diagnóstico y los que debutan con síntomas son clásicamente respiratorios (disnea, tos, hemoptisis o neumonia); $40 \%$ puede localizarse en la periferia y diagnosticarse como hallazgo radiológico incidental (3). Aunque los síntomas por producción hormonal son raros, el síndrome de Cushing se presenta en $2 \%$ de los carcinoides y son la causa más frecuente de producción ectópica de hormona de crecimiento (20). El sindrome carcinoide está presente en menos de $5 \%$ de los casos (21). Los hallazgos radiológicos incidentales son una forma importante de diagnóstico, en especial para las formas bien diferenciadas, por su curso indolente inicial con ausencia de síntomas o limitados a síntomas respiratorios.

Estos tumores bien diferenciados tienen el potencial de hacer metástasis, las cuales, en ciertos casos, son las que motivan el diagnóstico. La presencia de metástasis al diagnóstico variará de acuerdo con el espectro, encontrando tasas bajas en los pacientes con CT y más altas en los CA. En una serie de 142 casos en Israel con 128 casos de CT, 17 sujetos (13\%) cursaban con metástasis ganglionares y $2(1,6 \%)$ sujetos a distancia en hígado, a la vez que de los 14 sujetos con CA 6 tenian metástasis ganglionares (43\%) y $3(21 \%)$ a distancia en higado, bazo y cerebro (14). 
Por su parte, una serie española reúne 361 casos de tumores neuroendocrinos de pulmón de 1980 a 1997 con 261 CT y 43 CA con porcentaje de metástasis ganglionares de $4 \%$ y $23 \%$ respectivamente y a distancia de $1,5 \%$ y $20,9 \%$ (15).

En nuestra serie de los $26 \mathrm{CT}$ con datos clínicos, la mitad de los casos fueron diagnosticados como un hallazgo radiológico incidental mientras que la otra mitad presentó alguna sintomatologia que motivó el diagnóstico. En este caso la clínica se limitaba a sintomas respiratorios como tos, disnea, neumonia obstructiva, entre otros. En cuanto a las metástasis, $11 \%$ de los casos con datos presentaba metástasis, todos a nivel de ganglios regionales.

Para los CA se halló algo muy similar en la distribución de la presentación clínica con $50 \%$ de los casos asintomáticos y $50 \%$ con alguna manifestación clínica que en la mayoría fue respiratoria a excepción de un paciente con síndrome medular a causa de metástasis. El $62,5 \%$ de los pacientes con datos cursaba con diseminación de la enfermedad, de los cuales $40 \%$ la presentaba a nivel de ganglios regionales y $60 \%$ a distancia.

Consideramos que los aspectos clínicos y la presencia de metástasis es congruente con lo reportado en la literatura y se debe tener presente en todo momento ante este diagnóstico, ya que a pesar de ser tumores bien diferenciados, no son completamente indolentes, y la agresividad y el potencial de metástasis aumenta a medida que avanza el espectro tumoral.

De los tumores mal diferenciados, el CCP puede debutar con diferentes tipos de manifestaciones, ya sean constitucionales, pulmonares, sintomas por diseminación o desórdenes paraneoplásicos, siendo los más frecuentes fatiga, tos, disnea, disminución del apetito, pérdida de peso, dolor o hemoptisis o síntomas secundarios a metástasis extratorácicas; se puede encontrar el sindrome de vena cava hasta en el $10 \%$ de los sujetos (3). Estos tumores tienen gran potencial de producción hormonal, y de esta manera generan diferentes endocrinopatías. El sindrome de secreción inadecuada de ADH se presenta en $5,5 \%$ de los pacientes al momento del diagnóstico (18). El carcinoma neuroendocrino de célula grande usualmente es de localización periférica en $67 \%$ a $84 \%$ de los casos, llevando principalmente a síntomas torácicos no pulmonares por compromiso a estructuras vecinas o a sintomatologia dada por metástasis $(3,15)$.

Estos carcinomas de alto grado tienen un gran potencial metastásico desde el inicio. Hay presencia de metástasis extratorácicas a lugares como hueso, cerebro, hígado y adrenales en la mayoria de los pacientes al momento de la presentación (3). La serie española muestra que de 35 sujetos con CCP $20 \%$ tenia metástasis ganglionares y $37,1 \%$ a distancia, y de los 22 CNECG $41 \%$ cursaba con metástasis ganglionares y $63 \%$ con metástasis a distancia (15). Por su parte, una serie danesa con 11.998 pacientes con CCP mostró que $50 \%$ de los pacientes tenía enfermedad a distancia y $25 \%$ regional en ganglios, con $11 \%$ de pacientes sin datos reportados, mientras que de los 50 CNECG $40 \%$ tenía metástasis a distancia y $26 \%$ enfermedad en ganglios regionales, con $14 \%$ de pacientes sin información (13).

En nuestra serie, de los 14 sujetos con CCP de los que se contaba con información con respecto a la forma del diagnóstico, en la mayoria de los casos éste fue motivado por las manifestaciones clínicas secundarias al tumor, siendo las más frecuentes los sintomas respiratorios, pero otros como el sindrome de vena cava, la pérdida de peso, las alteraciones neurológicas y el dolor crónico y un caso de SIADH también figuraban como parte de la clínica. La mayoria de los pacientes cursaba con metástasis al momento del diagnóstico ( $98 \%$ de los sujetos con datos), principalmente a distancia. Sólo hay 2 casos de CNECG, por lo que no consideramos suficiente para hacer un análisis.

La principal debilidad de este trabajo es la pérdida de algunos datos debido a su condición de estudio retrospectivo, de los cuales muchos son diagnosticados en la Institución pero continúan seguimiento externo. Igualmente, no se pudo obtener la incidencia por no contar con las estadisticas globales de los tumores de pulmón y otros tumores en el HUFSFB. Aun no hay datos nacionales de incidencia y supervivencia de este tipo de tumor.

Con este estudio se busca motivar la investigación en este campo y alertar sobre el seguimiento necesario aun en los tumores bien diferenciados, que no son indolentes.

\section{BIBLIOGRAFIA}

1. Clark OH, Benson AB 3rd, Berlin JD, Choti MA, Doherty GM Engstrom PF, et al. NCCN Clinical Practice Guidelines in Oncology: neuroendocrine tumors. J Natl Compr Canc Netw. 2009; 7 (7): $712-47$

2. Chong S, Lee KS, Chung MJ, Han J, Kwon OJ, Kim TS. Neuroendocrine tumors of the lung: clinical, pathologic, and imaging findings. Radiographics. $2006 ; 26$ (1): 41-57.

3. Travis WD. Advances in neuroendocrine lung tumors. Ann Oncol. 2010; 21 (Supp| 7): VII65-71 
4. Bertino EM, Confer PD, Colonna JE, Ross P, Otterson GA. Pulmonary neuroendocrine/carcinoid tumors: a review article. Cancer. 2009; 115 (19): 4434-41.

5. Travis WD, Brambilla E, Muller-Hermelink HK, Harris CC. World Health Organization classification of tumours, pathology \& genetics, tumours of the lung, pleura, thymus and heart. Lyon, France, IARC Press, 2004.

6. Rekhtman N. Neuroendocrine tumors of the lung: an update. Arch Pathol Lab Med. 2010; 134 (11): 1628-38.

7. Ferolla P, Daddi N, Urbani M, Semeraro A, Ribacchi R, Giovenali $P$, et al. Tumorlets, multicentric carcinoids, lymph-nodal metastases, and long-term behavior in bronchial carcinoids. J Thorac Oncol. 2009; 4 (3): 383-7.

8. Righi L, Volante M, Rapa I, Scagliotti GV, Papotti M. Neuro-endocrine tumours of the lung. A review of relevant pathological and molecular data. Virchows Arch. 2007; 451 (Suppl 1): S51-9.

9. Modlin IM, Lye KD, Kidd M. A 5-decade analysis of 13,715 carcinoid tumors. Cancer. 2003; 97 (4): 934-59.

10. Taal BG, Visser O. Epidemiology of neuroendocrine tumours. Neuroendocrinology. 2004; 80 (Suppl 1): 3-7.

11. Oberg K, Hellman P, Kwekkeboom D, Jelic S. ESMO Guidelines Working Group. Neuroendocrine bronchial and thymic tumours: ESMO Clinical Practice Guidelines for diagnosis, treatment and follow-up. Ann Oncol. 2010; 21 (Suppl 5): v220-2.

12. Ferolla $\mathrm{P}$, Faggiano A, Avenia N, Milone F, Masone S, Giampaglia $F$, et al. Epidemiology of non-gastroenteropancreatic (neuro) endocrine tumours. Clin Endocrinol (Oxf). 2007; 66 (1): 1-6.

13. Skuladottir $H$, Hirsch FR, Hansen $H H$, Olsen JH. Pulmonary neuroendocrine tumors: incidence and prognosis of histolo- gical subtypes. A population-based study in Denmark. Lung Cancer. 2002; 37 (2): 127-35.

14. Fink G, Krelbaum T, Yellin A, Bendayan D, Saute M, Glazer M, et al. Pulmonary carcinoid: presentation, diagnosis, and outcome in 142 cases in Israel and review of 640 cases from the literature. Chest. 2001; 119 (6): 1647-51.

15. Garcia-Yuste M, Matilla JM, Alvarez-Gago T, Duque JL, Heras $F$, Cerezal LJ, et al. Prognostic factors in neuroendocrine lung tumors: a Spanish Multicenter Study. Ann Thorac Surg. 2000; 70 (1): 258-63.

16. Travis WD. Lung tumours with neuroendocrine differentiation. Eur J Cancer. 2009; 45 (Suppl 1): 251-66.

17. Hage R, de la Rivière AB, Seldenrijk CA, van den Bosch JM. Update in pulmonary carcinoid tumors: a review article. Ann Surg Oncol. 2003; 10 (6): 697-704.

18. Gustafsson BI, Kidd M, Chan A, Malfertheiner MV, Modlin IM. Bronchopulmonary neuroendocrine tumors. Cancer. 2008; 113 (1): 5-21.

19. Asamura $H$, Kameya $T$, Matsuno $Y$, Noguchi $M$, Tada $H$, Ishikawa $Y$, et al. Neuroendocrine neoplasms of the lung: a prognostic spectrum. J Clin Oncol. 2006; 24 (1): 70-6.

20. Pinzón B, Palacios D, García-Herreros LG, Acero R, Pavía J, Roa J, et al. Tumor carcinoide broncopulmonar. Rev Colomb Radio. 2006; 17 (2): 1942-1948.

21. Barreto J, Mejía B, Plata R, Lizacno J, González H, Serrano R. Broncoscopia: imagen y discusión. Tumor carcinoide endobronquial: resección endoscópica por electrocirugia. Rev Colomb Neumol. 2008; 20 (3): 107-110. 
alrededor del organismo (13). El diagnóstico en los dos casos reportados se basó en el análisis bioquími$\mathrm{co}$, histológico y microbiano de la muestra obtenida.

No hay un protocolo estandarizado para el tratamiento de la infección criptococócicà en ninguna localización anatómica específica, a excepción del pulmón y del sistema nervioso central. La Sociedad de Enfermedades Infecciosas de América (IDSA) 2010, en sus directrices recomienda que aquellos pacientes que no tienen compromiso meníngeo ni pulmonar y presentan una infección limitada a un solo sitio, pueden ser tratados con fluconazol ( $400 \mathrm{mg}$ [6 mg/kg] por dia por vía oral) durante seis a doce meses (recomendación B-III nivel) (14). La selección del agente antimicótico y la duración del tratamiento dependen de factores como la severidad de la enfermedad, el estado inmune del huésped y la respuesta terapéutica. Murphy y
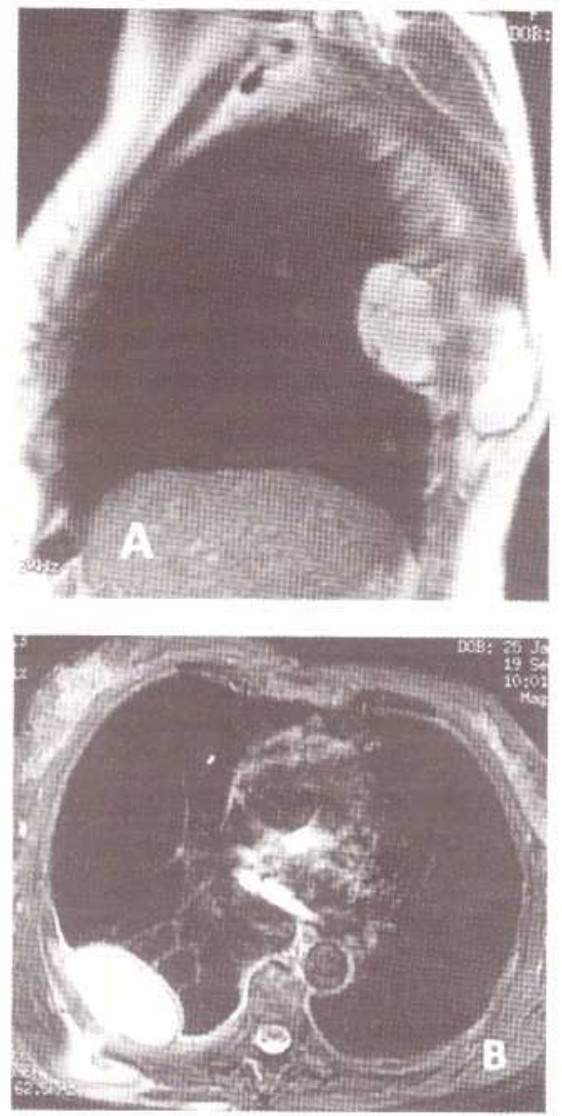

Figura 1. Resonancia magnética del tórax. Imagen coronal eco de espín con información T2 (A) e imagen axial con información T2, obtenida de una secuencia eco de espín con pulso de inversión recuperación/STIR (B). Se demuestra la presencia de una masa extrapulmonar marcadamente hiperintensa en el aspecto posterior del hemitórax derecho, compatible con una colección pleural loculada, con extensión asociada a los tejidos blandos subyacentes de la pared torácica. colaboradores publicaron la resolución completa de la osteomielitis en un solo sitio por infección criptococó cica después de diez semanas de fluconazol en uné dosis de $200 \mathrm{mg} / \mathrm{dia}$ (15).

\section{REPORTE DE CASOS}

\section{Caso 1}

Paciente de género femenino, de 68 años, quier presentó cuadro clínico de cuatro meses de evoluciór conformado por dolor progresivo localizado en la pa. red posterior del tórax derecho, no asociado a trauma el cual incrementaba con la movilización de la extre. midad superior derecha y estaba acompañado de pérdida de $6 \mathrm{~kg}$ de peso. Al examen físico se encontrć masa dolorosa a la palpación de $10 \times 9 \mathrm{~cm}$ de diáme. tro (Figuras 1 y 2 ).
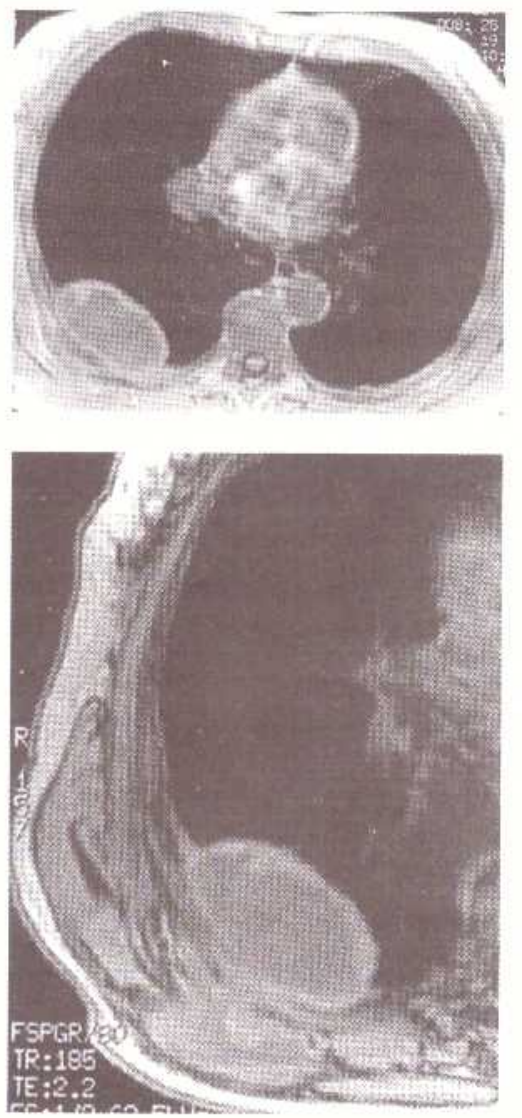

Figura 2. Resonancia magnética del tórax. Imágenes axiales eco de gradiente con información T1 post contraste $(A$, B). Las imágenes confirman la presencia de una colección pleural loculada en el aspecto posterior del hemitórax derecho, la cual presenta una extensión a los tejidos blandos subyacentes de la pared torácica. Se nota un engrosamiento asociado de la pleura parietal y visceral. 


\section{Caso 2}

Paciente femenina de 35 años con cuadro clínico de dos meses de evolución, consistente en masa dolorosa de $6 \mathrm{~cm}$ de diámetro, de crecimiento progresivo en la pared anterior del tórax derecho con erosión costal. Se realizó biopsia que reportó material fibrinopurulento. Ingresó a la institución donde realizaron resección local de la lesión de la pared torácica con reconstrucción con colgajo muscular (Figuras 3 a 5).

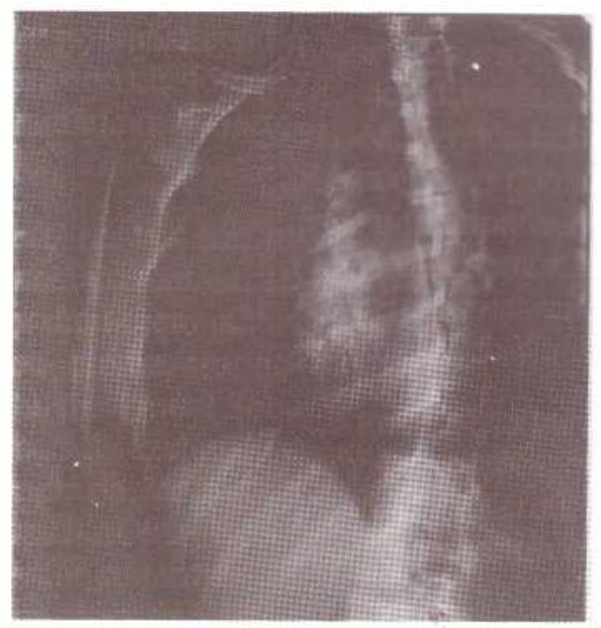

Figura 3. Radiografia de tórax. La proyección anterior oblicua derecha demuestra un aumento en la densidad de los tejidos blandos de la pared torácica, lo cual sugiere la presencia de una masa en esta localización.
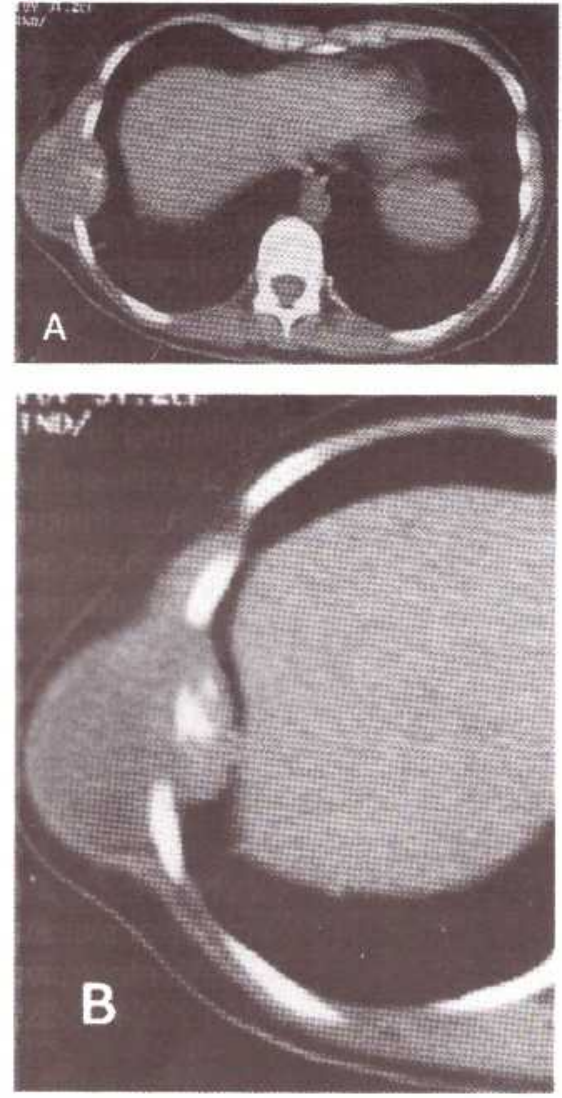

Figura 4. Escanografia simple del tórax (A, B). Las imágenes obtenidas en el plano axial demuestran la presencia de una masa bien definida, de apariencia sólida y de localización extrapulmonar en el aspecto lateral derecho de la pared torácica. Existe compromiso osteolitico costal subyacente.
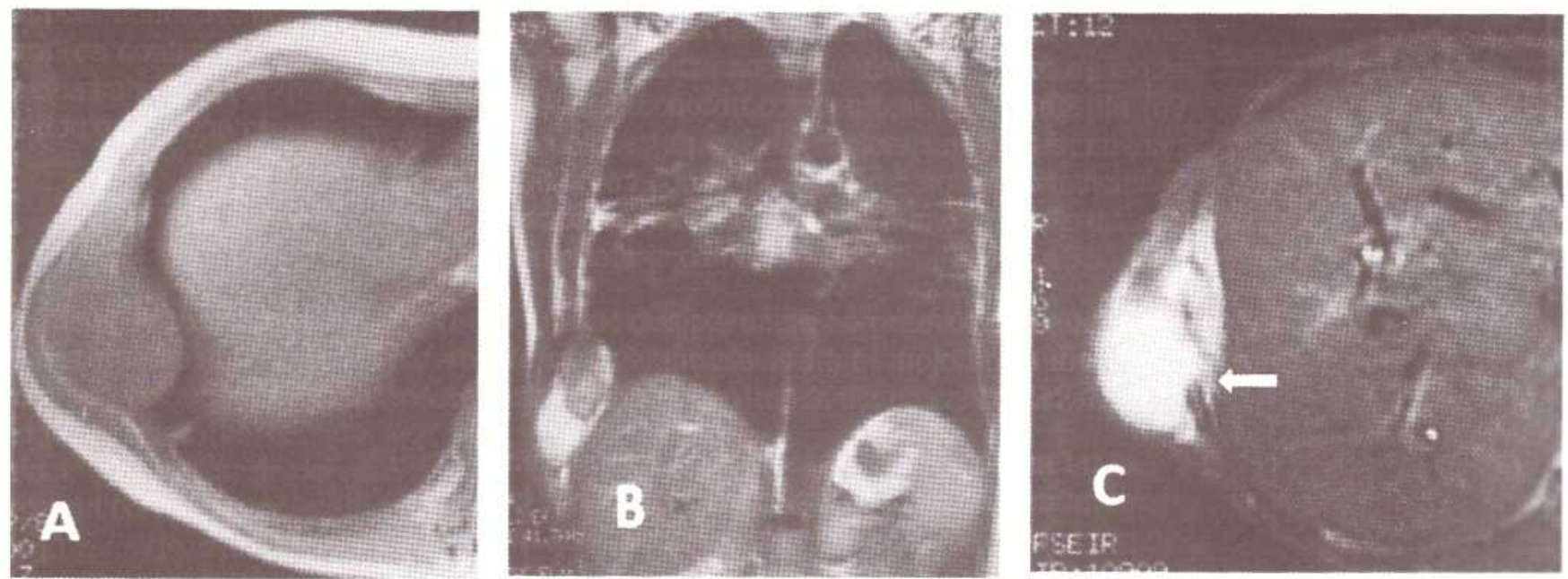

Figura 5. Resonancia magnética del tórax. Imagen axial con información T1 de una secuencia eco de gradiente (A) e imágenes con información T2 obtenidas de secuencias eco de espin en los planos coronal y axial (B, C). Se demuestra la presencia de una masa extrapulmonar sólida y bien definida en el aspecto lateral derecho de la pared torácica, con compromiso infiltrativo costal asociado (ver flecha). La lesión es de señal homogénea e isointensa con los músculos adyacentes en la imagen con información T1 (A) y presenta una señal heterogénea en las imágenes con información T2 (B, C). 


\section{BIBLIOGRAFÍA}

1. Lizarazo J, Linares M, De Bedout C, Restrepo A. Estudio clínico y epidemiológico de la criptococosis en Colombia: resultados de nueve años de la encuesta nacional, 1997-2005. Biomédica. 2007; 27:94-109.

2. Uribe P, Restrepo A, Diaz G. Estudio prospectivo clinico y microbiológico de las meningitis subagudas y crónicas. Antioquia Médica. 1973; 23: 153-64.

3. Lizarazo J, Mendoza M, Palacios D, Vallejo A, Bustamante A, Ojeda $\mathrm{E}$ et al. Criptococosis ocasionada por Cryptococcus neoformans variedad gattii. Acta Med Colomb. 2000; 25: 171 8.

4. Castañeda E, Torrado E, Arango M, de Bedout C, Tobón AM, Restrepo A et al. Criptococosis en Colombia: estudio interinstitucional. Inf Quinc Epidemiol Nac. 2000; 5: 115-9.

5. Góngora LR. Informe evento VIH/SIDA Semana epidemiológica 1 a 14, primer semestre julio 2005. Julio 13 de 2005. (Agosto 4 de 2006). Disponible en: http:// www.ins.gov.co/pdf/vcsp/ informe_evento_vih_junio_1sem2005.pdf.

6. Dromer F, Mathoulin S, Dupont B, Laporte A. Epidemiology of cryptococcosis in France: a 9-year survey (1985-1993). French Cryptococcosis Study Group. Clin Infect Dis. 1996; 23: 82-90.

7. Hakim JG, Gangaidzo IT, Heyderman RS, Mielke J, Mushangi $E$, Taziwa $A$, et al. Impact of HIV infection on meningitis in
Harare, Zimbabwe: a prospective study of 406 predominantly adult patients. AIDS 2000; 14: 1401-7.

8. Rozenbaum R, Goncalvez AJ. Clinical epidemiological study of 171 cases of cryptococcosis. Clin Infect Dis. 1994; 18: 369-80.

9. Zhang $Y$ et al. Cryptococcal osteomyelitis of the scapula and rib in an immunocompetent patient. Medical Mycology Month. 2012, Early Online: 1-5.

10. Al-Tawfiq JA, Ghandour J. Cryptococcus neoformans abscess and osteomyelitis in an immunocompetent patient with tuberculous lymphadenitis. Infection. 2007; 35: 377-382.

11. Behrman RE, Masci JR, Nicholas P. Cryptococcal skeletal infections: Case report and review. Rev Infect Dis. 1990; 12: 181-90.

12. Zach TL, Penn RG. Localized cryptococcal osteomyelitis in an immunocompetent host. Pediatr Infect Dis. 1986; 5: 601-603.

13. Perfect JR. Cryptococcus neoformans. In: Mandell GL, Bennett, Dolin R, editors. Mandell, Douglas, and Qadir et al. - Isolated cryptococcal osteomyelitis. J Infect Dev Ctries. 2011; 5 (9); 669-673.

14. Perfect JR, Dismukes WE, Dromer F, Goldman DL. Graybill JR, Hamill RJ, et al. Clinical practice guidelines for the management of cryptococcal disease: 2010 update by the Infectious Diseases Society of America. Clin Infect Dis. 2010; 50: 291-322.

15. Murphy SN, Parnell N. Fluconazole treatment of cryptococcal rib osteomyelitis in an HIV-negative man. A case report and review of the literature. J Infect. 2005; 51: e309-311. 